\title{
ASSOCIATION BETWEEN CRYOGLOBULINEMIA AND THYROID DYSFUNCTION IN HEPATITIS C CARRIERS
}

\author{
Luís Jesuíno de Oliveira Andrade*, Ajax Mercês Atta**, Maria Luiza Brito de Sousa Atta***, \\ Raymundo Paraná****
}

Corresponding author: Luís Jesuíno de Oliveira Andrade - luis_jesuino@yahoo.com.br

* PhD, Adjunct professor Health Department, State University Santa Cruz, Ilhéus, Bahia, Brazil

** PhD, Head Professor, School of Pharmacy, Federal University of Bahia, Brazil

*** PhD, Adjunct professor Department of Toxicological and Clinical Analysis, School of Pharmacy, Federal University of Bahia, Brazil

**** PhD, Associate Professor, Federal University of Bahia, Gastro-Hepatology Service, Federal University of Bahia, Brazil

\begin{abstract}
Introduction: Cryoglobulinemia is a biomarker of autoimmunity correlated with hepatitis $\mathrm{C}$ virus (HCV) infection. Objective: Report the association between the presence of mixed-cryoglobulinemia and thyroid dysfunction (TD) in hepatitis $C$ virus carriers before and during treatment with pegylatedinterferon-alpha (Peg-IFN- $\alpha$ ). Methods: Prospective cohort study. Results: Sixty-five untreated HCV patients, 38 women and 27 men were included in this study. Mean age of patients was $49.61 \pm 11.83$ years. Cryoglobulinemia was demonstrated in $24(36.92 \%)$ patients. The TD was demonstrated in 2 patients $(3.10 \%)$ before treatment with IFN- $\alpha$, in 4 patients $(6.20 \%)$ at 12 th week, and 8 patients $(12.30 \%)$ at 24 th week; whereas autoimmune thyroid (TPO-Ab) positive was demonstrated in 3 patients (4.60\%) before treatment, in 7 patients (10.80\%) at 12th week, and 12 patients $(18.50 \%)$ at 24 th week. In the sample analyzed, the risk of a patient with cryoglobulinemia develop TD was 2.74 ( $95 \%$ CI: $1.95-$ 12.95) times that of a patient without cryoglobulinemia in the 12th week of treatment and $1.83(95 \% \mathrm{Cl}$ : 1.03-7.08) times in the 24th week of antiviral treatment, and risk of a patient with cryoglobulinemia for develop TPO-Ab positive was 2.44 (95\% Cl: $1.46-5.98)$ times that of a patient without cryoglobulinemia in the 12th week of treatment, and 1.31 ( $95 \% \mathrm{Cl}: 1.05-2.89$ ) times in the 24 th weeks of antiviral treatment. Conclusion: We concluded that thyroid function evaluation should be recommended for HCV carriers that are seropositive for cryoglobulinemia
\end{abstract} Keywords: Hepatitis C; Interferon-alpha; Thyroid diseases; Cryoglobulinemia. 


\section{INTRODUCTION}

Thyroid dysfunction (TD) is one of the commonest endocrine manifestations of chronic hepatitis $C$ infection (HCV), exacerbated by pegylated interferon-alpha (Peg-IFN- $\alpha$ ) treatment. $^{(1)}$ Mixed cryoglobulinemia (MC) is a systemic vasculitis was characterised by the deposition of circulating immuno-complexes in small and medium-sized blood vessels resulting in a variety of clinical manifestations. A study showed that HCV infection associated with $\mathrm{MC}$ is accompanied by autoimmune thyroiditis and TD, usually hypothyroidism. ${ }^{(2)}$

IFN- $\alpha$ therapy is associated with the development of TD and/or thyroid autoimmunity due to its effects on the immune system, by an immunomodulatory mechanism, and also by direct action on the thyroid.(3) The genetic susceptibility may explain the increased prevalence of autoimmune thyroid disorders in MC patients. ${ }^{(4)}$ Morphological evidence of hepatitis $C$ virus replication in thyroid cells in immune competent patients has still not been demonstrated. The objective of this study was to identify the frequency of the autoimmune changes, and assess the association between the presence of cryoglobulinemia and TD in hepatitis $C$ virus carriers before and during treatment with Peg-IFN- $\alpha$.

\section{PATIENTS AND METHODS}

\section{STUDY DESIGN}

Type of study: prospective cohort study. Cryoglobulinemia was assessed only prior to commencement of treatment (Mo). The TD was investigated in all patients by tests that included thyroid stimulating hormone (TSH), free thyroxine ( $\mathrm{FT}_{4}$ ), and TPO-Ab, before initiating treatment (Mo), at week $12\left(\mathrm{M}_{3}\right)$ and at the end week 24 of treatment (M6). Measurements of TRAb were only performed in patients who had clinical and laboratorial hyperthyroidism.

\section{PATIENTS}

Sixty-five HCV carriers ( 38 women and 27 men), with a mean age of $49.61 \pm 11.83$ years (ranging from 20 to 67 years), from one hepatitis reference center in Salvador (Bahia, Brazil) were included in this study. They tested positive for serum anti-HCV antibodies by enzyme-linked immunosorbent assay with structural and non-structural HCV antigens (AXSYM System; Abbott Laboratories, Chicago, IL, USA) and for HCV-RNA by polymerase chain reaction (PCR) (AmplicorTM HCV Detection KIT V2.0; Roche Molecular Systems Inc., Somerville, NJ, USA). Forty-five carriers were infected with $\mathrm{HCV}$ genotype 1 , and 20 with the genotype 3 as determined by the INNOLIPA test (Innogenetics, NV, Gent, Belgium).

The study protocol was approved by the Ethics Committee of University State of Santa Cruz, Bahia, Brazil (protocol 166/08 - 326), in accordance with the Declaration of Helsinki. All subjects gave their informed consent to go into the study.

\section{CRYOGLOBULINEMIA ASSESSMENT}

Cryoglobulins was investigated using both tube and gel cryoprecipitation. (5) Cryoglobulins were fractionated on an agarose gel (Paragon Serum Protein Electrophoresis Kit, Beckman Coulter, Inc., Fullerton, CA, USA), and probed with antisera to immunoglobulin for the heavy and light-chain determinants (Dako A/S, Glostrup, Denmark).

\section{THYROID FUNCTION AND ANTIBODY THYROID SCREENING}

$\mathrm{TSH}$ (ultra sensitive third-generation method with a reference normal range of $0.35-5.50 \mathrm{mIU} / \mathrm{L}$ ), and $\mathrm{FT}_{4}$ (reference normal range of $0.58-1.40 \mathrm{ng} / \mathrm{dL}$ ) were assayed using commercially available kits by immunometric assays.

Euthyroidism is defined as TSH and $\mathrm{FT}_{4}$ within the normal reference range while not taking any thyroid medication. Hypothyroidism is biochemically was 
characterized by low or normal $\mathrm{FT}_{4}$ concentrations with $\mathrm{TSH}$ above its upper limit. Hyperthyroidism is defined by TSH below its lower reference limit with high or normal $\mathrm{FT}_{4}$ concentrations. While, hypothyroxinemia is characterized by TSH between it reference intervals with low $\mathrm{FT}_{4}$ concentration.

TPO-Ab was detected by solid phase 2-site sequential chemiluminescent immunometric assay (normal: < $40 \mathrm{IU} / \mathrm{mL}$ ). Patients were classified as positive for thyroid autoimmunity with $T P O-A b \geq 40$ $\mathrm{IU} / \mathrm{mL}$, and negative with TPO-Ab $<40 \mathrm{IU} / \mathrm{mL}$.

\section{STUDY OUTCOMES}

The primary outcome of this study was the relative risk of development of TD and autoimmune thyroiditis in those patients with positive cryglobulimemia in $M_{3}$ and $M 6$ of treatment.

\section{STATISTICAL ANALYSIS}

Data are expressed as mean \pm SD for those variables that were normally distributed. Statistical analysis was performed using chi-square or Fischer's exact test. Relative risk was calculated by using the onetailed exact method for the combination of $2 \times 2$ tables, computing 95\% confidence intervals $(\mathrm{Cl})$.

Statistical analysis was performed using SPSS $\mathbb{R}$ version 16.0 for Windows (SPSS Inc, Chicago, III), and a p-value $<0.05$ was considered statistically significant.

\section{RESULTS}

Mean age of the 65 patients ( 38 females and 27 males) was $49.61 \pm 11.83$ years (range: $22-66$ years), with 46 patients with Genotype 1, 1 patient genotype 2 , and 18 patients genotype 3 . The demographic data are present in Table 1.

Table 1 - Demographic data in studied group

\begin{tabular}{|c|c|c|c|c|c|c|c|}
\hline $\mathrm{N}$ & AGE & GENDER* & GENOTYPE & $\mathrm{N}$ & AGE & GENDER & GENOTYPE \\
\hline 01 & 30 & $F$ & 1 & 34 & 25 & $F$ & 1 \\
\hline 02 & 49 & $F$ & 1 & 35 & 52 & $M$ & 3 \\
\hline 03 & 55 & F & 1 & 36 & 41 & $M$ & 1 \\
\hline 04 & 48 & F & 1 & 37 & 54 & $\mathrm{~F}$ & 2 \\
\hline 05 & 63 & $\mathrm{~F}$ & 3 & 38 & 53 & F & $\overline{1}$ \\
\hline 06 & 57 & $\mathrm{~F}$ & 1 & 39 & 46 & $M$ & 1 \\
\hline 07 & 25 & $M$ & 1 & 40 & 24 & $M$ & 1 \\
\hline 08 & 57 & F & 1 & 41 & 66 & $M$ & 1 \\
\hline 09 & 50 & $M$ & 3 & 42 & 60 & $\mathrm{~F}$ & 1 \\
\hline 10 & 52 & $M$ & 1 & 43 & 59 & $M$ & 1 \\
\hline 11 & 66 & $F$ & 3 & 44 & 59 & $M$ & 1 \\
\hline 12 & 51 & $M$ & 3 & 45 & 50 & $\mathrm{~F}$ & 1 \\
\hline 13 & 29 & $M$ & 1 & 46 & 45 & $M$ & 1 \\
\hline 14 & 30 & $\mathrm{~F}$ & 3 & 47 & 54 & $\mathrm{~F}$ & 1 \\
\hline 15 & 63 & $F$ & 3 & 48 & 64 & $F$ & 1 \\
\hline 16 & 54 & $F$ & 3 & 49 & 47 & $M$ & 1 \\
\hline 17 & 30 & $\mathrm{~F}$ & 1 & 50 & 62 & $\mathrm{~F}$ & 1 \\
\hline 18 & 22 & $\mathrm{~F}$ & 3 & 51 & 48 & $\mathrm{~F}$ & 3 \\
\hline 19 & 49 & $F$ & 1 & 52 & 49 & $M$ & 1 \\
\hline 20 & 54 & $M$ & 1 & 53 & 58 & F & 1 \\
\hline 21 & 62 & $M$ & 1 & 54 & 58 & $F$ & 1 \\
\hline 22 & 49 & $F$ & 1 & 55 & 52 & $F$ & 3 \\
\hline 23 & 53 & $M$ & 3 & 56 & 59 & $M$ & 1 \\
\hline 24 & 35 & $F$ & 3 & 57 & 51 & $\mathrm{~F}$ & 1 \\
\hline 25 & 37 & $F$ & 1 & 58 & 56 & $M$ & 1 \\
\hline 26 & 54 & $M$ & 3 & 59 & 63 & $M$ & 3 \\
\hline 27 & 58 & $F$ & 1 & 60 & 48 & $F$ & 1 \\
\hline 28 & 25 & $M$ & 1 & 61 & 65 & F & 1 \\
\hline 29 & 58 & $F$ & 1 & 62 & 60 & $F$ & 1 \\
\hline 30 & 50 & $M$ & 3 & 63 & 36 & $F$ & 3 \\
\hline 31 & 56 & $F$ & 3 & 64 & 48 & $M$ & 1 \\
\hline 32 & 65 & $M$ & 1 & 65 & 34 & $\mathrm{~F}$ & 3 \\
\hline 33 & 46 & $M$ & 1 & & & & \\
\hline
\end{tabular}

* $\mathbf{M}=$ male $\mathbf{F}=$ female 


\section{CRYOGLOBULINEMIA, THYROID DYSFUNCTION, AND AUTOIMMUNE THYROID (M0)}

Baseline cryoglobulinemia was demonstrated in 24 (36.92\%) patients. The TD was demonstrated in 2 patients $(3.10 \%)$ before treatment with IFN- $\alpha$, whereas TPO-Ab positive was demonstrated in 3 patients $(4.60 \%)$ before treatment. The positivity of TPO-Ab before treatment was more found in patients with TD than those without thyroid disorders $(p=0.04)$. No significant association was observed among gender, age, or HCV genotypes, with thyroid disorders at baseline. Patients who had TD were treated upon diagnosis.

\section{THYROID DYSFUNCTION AND AUTOIMMUNE THYROID (M3)}

After $\mathrm{M}_{3}$ of treatment, 4 patients $(6.20 \%)$ presented TD, and 7 patients $(10.80 \%)$ presented TPO-Ab positive. One case of subclinical hypothyroidism that occurred during treatment was associated with TPO-Ab positivity. Statistical analysis showed that the risk of a patient with cryoglobulinemia develop TD was 2.74 (95\% Cl: 1.95-12.95) times that of a patient without cryoglobulinemia in the $\mathrm{M}_{3}$ of treatment, and risk of a patient with cryoglobulinemia for develop TPO-Ab positive was $2.44(95 \% \mathrm{Cl}: 1.46-5.98)$ times in the $\mathrm{M}_{3}$ of treatment. In the 11 cases with thyroid disorders at the end of the $\mathrm{M}_{3}$ of treatment, were including the cases before treatment with IFN- $\alpha$.

\section{THYROID DYSFUNCTION AND} AUTOIMMUNE THYROID (M6)

At the end of M6 of treatment, 8 (12.30\%) patients developed TD, and 12 patients (18.46\%) presented TPO-Ab positive. Therefore, 14 cases of thyroid disease occurred at the end of M6 of treatment (21.53\%), being 7 cases of TD, 4 individuals had subclinical hypothyroidism, and 3 subclinical hyperthyroidism $(p=0.006)$. Additionally, 12 (18.46\%) patients with positive TPO-Ab $(p=0.002)$ were diagnosed at the end of $M 6$ treatment, including the patients in baseline (before), and $\mathrm{M}_{3}$ of treatment with IFN- $\alpha$.

The relative risk (RR) of developing TD was higher among those who developed the thyroid autoimmunity in patients with cryoglobulinemia positive at M6.

In the sample analyzed the risk of a patient with cryoglobulinemia develop TD was $1.83(95 \% \mathrm{Cl}$ : 1.03-7.08) times in the M6 of antiviral treatment, and risk of a patient with cryoglobulinemia for develop TPO-Ab positive was 1.31 (95\% Cl: $1.05-$ 2.89) times in the $M 6$ of antiviral treatment.

The thyroid disease occurred more often in female patients aged above 40 years old, with a significant association $(p=0.009)$ when analyzed at M6 of therapy.

In order to organize the data including the correlations of study, the results are present in Table 2.

Table 2 - Sample characteristics, Cryoglobulinemia, thyroid dysfunction, and autoimmune thyroid (continued)

\section{PATIENTS}

(MEAN AGE 49.61 FFL 11.83 Y)

\section{Cryoglobulinemia Thyroid dYSFUnction AUtoimmUne thyroid}

\section{First Month (Mo)}

Positive

Negative

$24(36.92 \%)$

$41(63.08 \%)$
$2(3.10 \%)$

$4(4.60 \%)$

Third Month (M3)

Positive

Negative

Relative Risk
$63(96.9 \%)$

$61(95.40 \%)$ 
Table 2 - Sample characteristics, Cryoglobulinemia, thyroid dysfunction, and autoimmune thyroid

(conclusion)

\begin{tabular}{lccc}
\hline \multicolumn{1}{c}{ Patients } & Cryoglobulinemia & Thyroid dysfunction & Autoimmune thyroid \\
(Mean AGe 49.61 FFL 11.83 Y) & . & \\
\hline Sixth Month (M6) & - & $8(12.30 \%)$ & $12(18.46 \%)$ \\
Positive & - & $57(87.20 \%)$ & $53(81.54 \%)$ \\
Negative & & $1.83(95 \% \mathrm{Cl}: 1.03-7.08)$ & $1.31(95 \% \mathrm{Cl}: 1.05-2.89)$ \\
Relative Risk & &
\end{tabular}

\section{DISCUSSION}

This study reports the association between the presence of cryoglobulinemia and TD in hepatitis $C$ virus carriers before and during treatment with pegylated- IFN- $\alpha$. The total relative risk of TD and TPO-Ab at the end of M 6 antiviral therapy with IFN- $\alpha$ and cryoglobulinemia positive were important.

Cryoglobulinemia is the presence of abnormal proteins in the blood, and are a frequent finding in HCV-infected patients. ${ }^{(6)}$ The pathophysiology is complex and involves humoral immunity, B and $T$ cellular immunity, but not directly the virus itself.(7) Cryoglobulinemia is defined as a systemic vasculitis arising of intravascular immune complexes named cryoglobulins, with simultaneous involvement of immunoglobulins (IgC and IgM) in generating of cryoglobulins that can include partially monoclonal or totally polyclonal immunoglobulins, elapsing the term MC. ${ }^{(8)}$

Estimates of mixed cryoglobulinaemia prevalence in patients with HCV infection vary widely from 10 to $70 \%,(9)$ in this study our data is consistent with the literature.

The association between HCV infection and cryoglobulinemia may be explained by the fact that this virus infects mononuclear cells. The presence of cryoglobulin in serum has not always been associated with important clinical manifestations, however in our study there was a high frequency of TD. ${ }^{(10)}$ Our study demonstrated thyroid disease involvement in HCV-related patients with cryoglobulinemia, ranging from of TD (overt hypothyroidism and hyperthyroidism, subclinical hypothyroidism or hyperthyroidism) and TPO-ab. These findings are in agreement with previous studies reporting TD associated the cryoglobulinemia positive. ${ }^{(2,4)}$

Considering its complex etiopathogenesis and clinical polymorphism, the treatment of cryoglobulinemia is especially challenging. HCV infection represents the main causative factor of the disease by exerting a chronic stimulus on the immune-system. ${ }^{(11)}$ The most effective treatment for cryoglobulinemia in HCV infection is eradication of the underlying HCV infection, and the current standard is Peg-IFN- $\alpha$ plus ribavirin.

The TD the most common endocrinopathy exacerbated by Peg-IFN- $\alpha$ and ribavirin treatment. (12) However, the mechanisms of induced thyroid disease are complex and not completely understood. The clear association between autoimmune thyroid disease and IFN- $\alpha$ use suggests that high endogenous IFN- $\alpha$ level may also be associated with naturally autoimmune thyroid disease. PegIFN- $\alpha$-induced autoimmune thyroid disease is associated with elevated auto-antibodies and may manifest with or without clinical disease. ${ }^{(13)}$ The relative risk of developing thyroid autoimmunity among HCV carriers treated with Peg-IFN- $\alpha$ and RIB was 5.8 times higher than the risk for developing TD. (1) Ours data showed a reduction of relative risk of TD and TPO-Ab at the end of M6 antiviral therapy with Peg-IFN- $\alpha$ and cryoglobulinemia positive, probably due to the reduction of viral load and consequent cryoglobulinemia reduction. A previous study described patients with HCV-induced 
cryoglobulinemia experienced complete remission of vasculitis symptoms accompanied by negative findings on serum viral load after treatment with antiviral agents. ${ }^{(14)}$ Several authors have reported the efficacy of Peg-IFN- $\alpha$ in the inhibition of HCV replication and reduction of cryoglobulin levels. The therapy with Peg-IFN- $\alpha$, as monotherapy, in cryoglobulinemia shows a complete response rate of $10-12 \%$ of cases. Complete response to therapy using a combination of Peg-IFN- $\alpha$ plus ribavirin varies in different studies from $18 \%$ to $64 \%$ of cases. ${ }^{(15)}$

Currently, only six studies have evaluated thyroid disorders in patients with cryoglobulinemia, and the results showed a higher prevalence of thyroid disorders in patients with cryoglobulinemia and $\mathrm{HCV}$ in comparison with controls and with $\mathrm{HCV}$ patients without cryoglobulinemia. ${ }^{(16)}$ This study suggests that cryoglobulinemia in HCV patients should be screened for TSH and TPO-Ab at the first evaluation. A careful follow-up of thyroid function should be conducted before and after treatment with Peg-IFN- $\alpha$, because beyond cryoglobulinemia and HCV, the Peg-IFN- $\alpha$ therapy presents known risk for the development of TD and TPO-Ab. In this way, prospective studies in this field are required to definitely confirm the association between cryoglobulinemia thyroid disorders, and HCV.

\section{CONCLUSION}

In conclusion, this study shows important clarification of the association between cryoglobulinemia positive in patients with $\mathrm{HCV}$ infection and thyroid disorders. Accordingly, some guidelines may be suggested as follows: patients with cryoglobulinemia positive who are treated with Peg-IFN- $\alpha$ and ribavirin therapy should be informed about the risks of developing thyroid dysfunction, and therefore screening of thyroid function, the panel of antibodies should be evaluated before and during treatment of HCV.

Conflict of interest: no potential conflict of interest relevant to this article was decl.

\section{REFERENCES}

1. Andrade LJ, Atta AM, Atta ML, Mangabeira $\mathrm{CN}$, Paraná R. Thyroid disorders in patients with chronic hepatitis $C$ using interferon-alpha and ribavirin therapy. Braz j infect dis. 2011;15:377-81.

2. Fallahi P, Ferrari SM, Giuggioli D, Corrado A, Fabiani S, Marchi S et al. Mixed cryoglobulinemia and thyroid autoimmune disorders. Clin Ter. 2013;164(4):e337-41.

3. Tomer Y, Blackard JT, Akeno N. Interferon alpha treatment and thyroid dysfunction. Endocrinol Metab Clin North Am. 2007;36:1051-66.

4. Antonelli A, Ferri C, Fallahi P, Giuggioli D, Nesti $\mathrm{C}$, Longombardo $\mathrm{G}$, et al. Thyroid involvement in patients with overt $\mathrm{HCV}$-related mixed cryoglobulinaemia. QJM. 2004;97:499-506.

5. Okazaki T, Nagai T, Kanno T. Gel diffusion procedure for the detection of cryoglobulins in serum. Clin Chem. 1998;44:1558-9.

6. Monti G, Saccardo F, Castelnovo L, Novati P, Sollima S, Riva A, et al. Prevalence of mixed cryoglobulinaemia syndrome and circulating cryoglobulins in a population-based survey: The Origgio study. Autoimmun Rev. 2014;13(6):60914.

7. Cacoub P, Sène D, Saadoun D. Cryoglobulinemia. Rev Med Interne. 2008:29:200-8.

8. Gragnani L, Fognani E, Piluso A, Zignego $A L$. Hepatitis $C$ virus-related mixed cryoglobulinemia: Is genetics to blame? World J Gastroenterol. 2013;19:8910-8915.

9. Charles ED, Dustin LB. Hepatitis C virus-induced cryoglobulinemia. Kidney Int. 2009;76:818-24.

10. Codes L, de Freitas LA, Santos-Jesus R, Vivitsk L, Silva LK, Trepo C et al. Comparative study of hepatitis $C$ virus genotypes 1 and 3 in Salvador, Bahia Brazil. Braz j infect dis. 2003;7:409-17.

11. Landau DA, Saadoun D, Halfon P, MartinotPeignoux M, Marcellin P, Fois E et al. Relapse of hepatitis $C$ virus-associated mixed cryoglobulinemia vasculitis in patients with sustained viral response. Arthritis Rheum. 2008;58:604-11.

12. Tran HA, Malcolm Reeves GE, Gibson R, Attia JR. Development of thyroid diseases in the 
treatment of chronic hepatitis $\mathrm{C}$ with alphainterferon may be a good prognosticator in achieving a sustained virological response: a meta-analysis. J Gastroenterol Hepatol. 2009;24:1163-8.

13. Mandac JC, Chaudhry S, Sherman KE, Tomer $Y$. The clinical and physiological spectrum of interferon-alpha induced thyroiditis: toward a new classification. Hepatology. 2006;43:661-72.

14. Levine JW, Gota C, Fessler BJ, Calabrese LH, Cooper SM. Persistent cryoglobulinemic vasculitis following successful treatment of hepatitis $C$ virus. J Rheumatol. 2005;32:1164-7.
15. Mazzaro C, Pozzato G, Zorat F, Baragiotta AM, Biscontin MG, Spina M, et al. Etiologic treatment of hepatitis $C$ virus-associated mixed cryoglobulinemia. Dig Liver Dis. 2007;39(Suppl 1):S1O2-6.

16. Fallahi P, Ferrari SM, Giuggioli D, Manfredi A, Mancusi C, Fabiani S, et al. Thyroid involvement in hepatitis $C$ - associated mixed cryoglobulinemia. Hormones (Athens). 2014;13:16-23 